\title{
Design and Implementation of a Tool for the Analysis and Management of Cardiac Parameters
}

\author{
Jorge Aguilera Perez, Rene Gonzalez Fernadez \\ Central Institute of Digital Research, Havana, Cuba
}

\begin{abstract}
The aim of this paper is to discuss the main features of a desktop application designed to study the evolution of heart diseases in the community based on the evolution of a group of electrocardiographic parameters. Periodically. Cornell and Sokolow indexes are computed to study ventricular hypertrophy; spatial dispersion of QT interval is calculated to predict malignant arrhythmias and the Selvester score is computed for persons who have suffer a myocardial infarction.

The Qt Creator platform, a SQL database engine and $\mathrm{C}++$ language were used to develop the proposed application. Several graphical tools were developed to study the ECG and for data representation. Also, a wide set of PDF reports can be generated or printed directly using a printer. A simple protocol designed by the authors is used in the communication process among Cuban ECG machines and the proposed application.

A final version of the application is being tested with fifteen patients in order to present it to medical registration in Cuba.
\end{abstract}

\section{Introduction}

Informatics and Technology growing impact on most of the human life scenes it is also present on the Medical field. This paper has been developed inside that scenery as a contribution to prevent the cardiac diseases occurrence on a community by doing a systemic study on the "still electrocardiographic signal (ECG)" in order to detect some pathological situations on early states. To achieve that goal the design of an application that fulfills the following requirements has been proposed:

- Reception and storage of the ECG wave and the measurements that has been done coming from the CardiHogar register equipment through a USB communication channel.

- Possibility of sending persons lists to been studied to the CardiHogar register equipment.
- Possibility of generating the following studies for each patient: Sokolow Index, Tacogram, Selvester's Score, QT Spatial Dispersion

- Graphic User interface that allows the ECG signal, measurements and trend graphics, visualization and analysis.

- Statistics visualization of the database content according to the following criteria: age groups and sex groups.

- Report generation using PDF format to be storage or printing.

- $\quad$ Report printing.

\section{Materials and methods}

\subsection{General description}

The developed application is included on an ECG system to study and to follow up patients with a potential risk to develop a cardiac affection whether is because they have risk factors such as obesity, hypertension among others or because they have already suffered a cardiac event such as a heart stroke. This system is formed by two main elements: the CardiHogar register device and the developed desktop application described in this paper. Communication between both components it is done through a USB communication channel and a communication protocol deigned by the authors, which allows the information interchange between the application and the device.

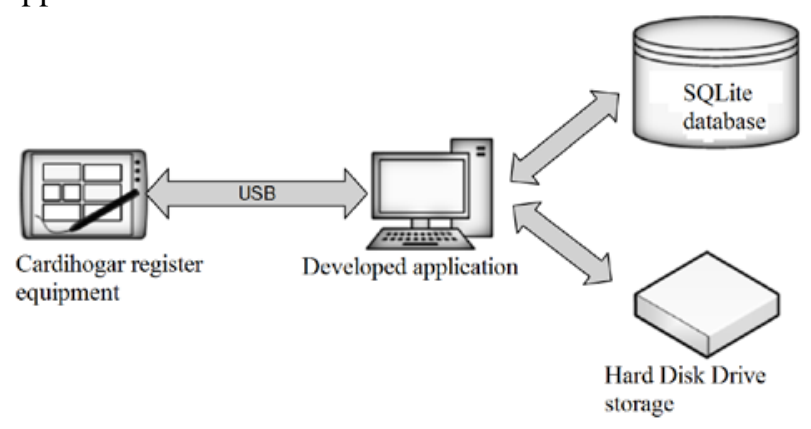

Figure 1. System general diagram 


\subsection{Development tools}

The Qt Creator development system, which is based on $\mathrm{C}^{++}$programming methods, has been selected to develop the application. This decision is based on the fact that the software that runs on the register device has been programmed using the same tool. This allows the interchange and code reutilization between both parts of the system reducing the development time because in both similar resources are used (data bases, ECG signal visualization, and so on). Also the development environment presents potent tools to an easily and time reduced development of graphic user interfaces always obtaining great results.

Application's data base has been implemented using the SQLite data base engine. It has been selected taking into account the minimum computational requirements demanded by this tool, what makes it a good choice to take into account when developing small and midcomplexity applications regarding to the data management. The data base design was done using the free distribution software SQLite Studio. This tool permits to design and implement the tables that conforms the application`s data base in a quick way.

\subsection{Application design}

The application design process is focused on the object oriented programming techniques and took into account a previous definition of the user roles of the actors that interacts with the system and the previously defined functionality. This perspective was determinant to select the Qt development platform since it is based on Object Oriented Programming. Inheritance, polymorphism and encapsulation are used as along the design of this application and also class composition techniques. Also, some new functionalities of the language has been used such as signals and slots to implement task synchronization. This is a mechanism that results novel on this work environment. Also the possibility of concurrent processing and non-synchronic events attention are used to achieve better results.

\subsection{User interface}

The user interface design bases all his potentiality on the resources available within the development tool, Qt environment. Layout's usage allowed one of the most important features achieved and is the fact that the application can be resized to any resolution always getting a rearranging of the user interface elements that permits the user to use the application without the need of scroll bars.

User interface elements distribution has been designed in a manner that the user can use it in an intuitive way and without the need of reading a user manual. Also a quick access to the functionalities is guaranteed.

On the left side there is an area in which the patient can be selected and also the study for which the information should be visualized.

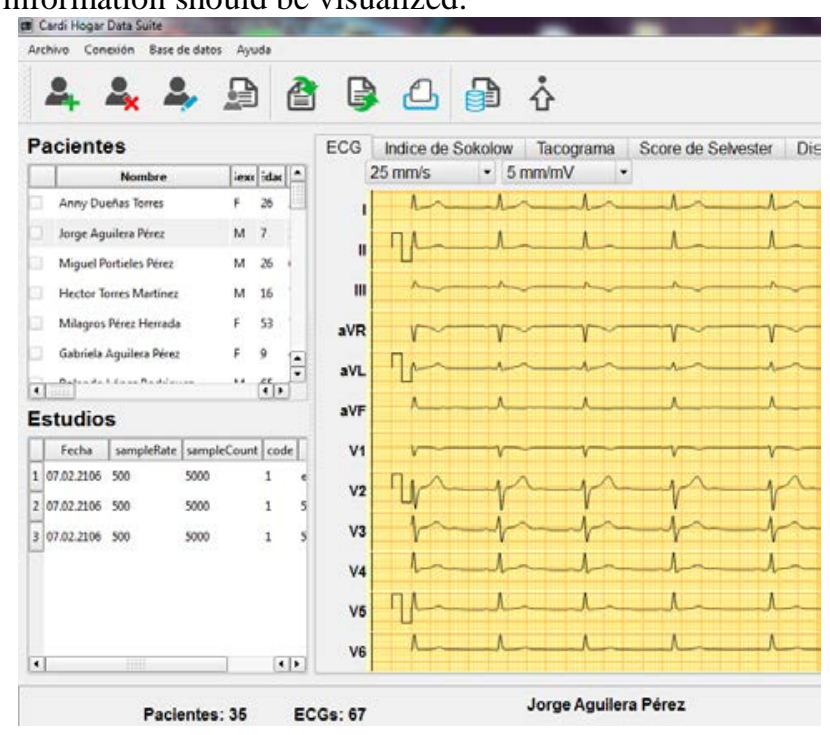

Figure 2. Main window

On the right side there is an area in which all the measurement and graphics are shown. This information has been divided using tabs that allow to change context quickly regarding to the information that is visualized. Further on this paper those tabs will be explained closely.

\subsection{ECG signal visualization}

\subsubsection{Speed and sensibility change}

The application allows to change speed and sensibility values, the typical values used for this kind of applications. Allowed values for sensibility are: 5, 10 and $20 \mathrm{~mm} / \mathrm{mV}$. Speed allowed values are: 12.5, 25 and 50 $\mathrm{mm} / \mathrm{s}$.

This functionality has been implemented on a way that differs a little from the usual way it is implemented.

It is common on applications of this kind that a change on any of the parameters (speed or sensibility) the signal is rescaled to make it fit the grid or how it is commonly known as signal image zoom. It is possible that when the signal is zoomed some of the data we are watching might disappear from the sight and it would be necessary to scroll the signal. In our application case is a non-desired effect since the signal should be always visible.

In the case of the developed application the grid is resized to fit the speed and sensibility settings keeping the signal invariant and always visible. This allows to always have the whole signal visible. 


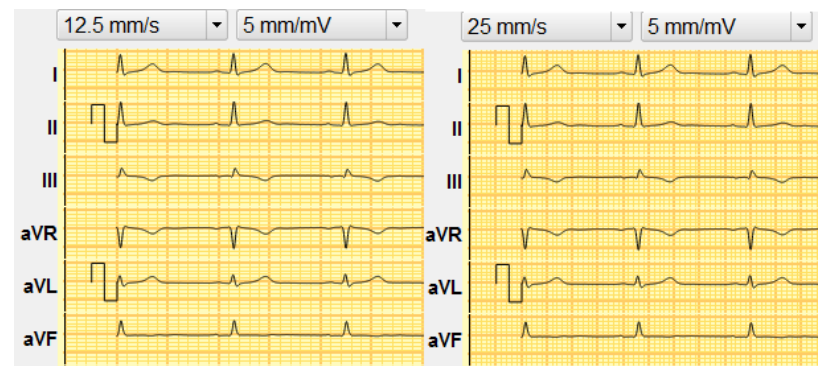

Figure 3. Speed change effects

\subsubsection{Visualization adjustment}

Electrocardiographic signals does not have the same amplitude among them or among different patients also varying on the same person on different stages of the day. This fact makes important to achieve that all the signals, no matter if they are small or big, are shown completely. The way implemented to solve this was to find the maximum and minimum values al across the signals to later stablish the graphic display limits. Doing this it is guaranteed that all the information will be shown without losing aspect ratio among the displayed signals.

\subsection{Studies}

\subsubsection{Sokolow index}

This is one of the most used indexes to study the Left Ventricular Hypertrophy, an affection that generates a great number of people with limitations to make physical efforts [2]. In order to analyze the associated information the screen is arranged as follows:

- The cardiac cycle in which the amplitude measurements of the QRS complexes is shown. The beginning and the end of the QRS complex is marked and also the beginning and the end of the PR and QT intervals respectively.

- All the Sokolow indexes values related to each one of the ECGs that are saved on the database for the selected patient. A trend line is drawn to show a prediction of the expected behavior on that parameter.

- A summary table can be found showing the PR, QRS and QT interval duration for each derivation.

\subsubsection{Tacogram}

It permits the study of the heart rate variations across the time, it makes it of great help as a complement to heart rate variability researches [3]. The information related to this study it is arranged as follows:
- The segment where the measurement was done is shown for all the derivations from DI to V6 arranged on groups of six. Also the ST segment deviation is available for each derivation.

- DII is completely shown, followed by a graphic in which the heart rates of each study related to the selected patient are represented. A trend line indicates the possible behavior of the parameter.

\subsubsection{Selvester score}

When a person suffers a heart stroke, a region of the heart is affected, but the extension of the region is not known until the cardiac muscle can be analyzed directly [4]. That is the reason why the Selvester score was conceived. It allows to stablish the magnitude of the affected area using a score system for it. The score is calculated using the measured QRS complex results. For the Selvester score study the information has been arranged as follows:

- The segment in which the measurements where made is shown. The derivations from DI to V6 are represented in groups of six.

- A summary table is shown containing the amplitude and duration for the $\mathrm{Q}, \mathrm{R}, \mathrm{R}^{\prime}$ and $\mathrm{S}$ expressed in $\mathrm{mV}$ and $\mathrm{ms}$ respectively.

- A table containing all the criteria applied to calculate the Selvester score and bold text has been used to remark the ones that are meet. On the bottom part of this table the score obtained is shown.

By using this method to show the results it is also possible to help professional training for those that do not know the way of calculating this score.

\subsubsection{QT interval spatial dispersion}

QT interval spatial dispersion, dQT from now on, is internationally recognized as a prognostic indicator of the sudden death [5]. Trending study for this parameter can be of great help before getting to a critic situation that could involve patient's life. To make an easy understanding of this study the information is arranged as follows:

- Segment in which the measurements where made is shown. The derivations from DI to V6 are represented in groups of six.

- DII derivation is shown completely to help in the calculation of the cardiac rhythm analysis.

- The values related to the selected patient are plotted. A trend line is also plotted to show the possible behavior of the parameter. 
- A summary table is shown, containing de QT and QT corrected duration information regarding to all the derivations.

\subsection{Communication}

The implemented protocol by the authors on the user side bases his functionality on the master/slave philosophy. The master role is adopted by the developed application and it is in charge of sending the acquisition and analysis requests to de medical device. The basic unit are the blocks and their basic composition is as follows:

- Block header

- Data

- Checksum

- Block End

Blocks are received one by one and always checked looking for data consistency using the checksum verification. If any anomaly is detected then a block retransmission is requested. This procedure is done a finite number of times before aborting the communication. The user is notified if such and error happens.

\subsection{Data base and information storage}

In order to relief the huge amount of data that represents the fact of storing all the signal information on the database it is decided to store only the basic information that allows to work with the potentialities of working with a data base. The remaining information is stored on the hard drive and it is only loaded into memory when it is needed. This way an optimum access to information and visualization is achieved.

Finally the database is composed by two tables: patients and studies. On the patient table the general patient information is stored.

On the studies table the data related to studies is stored. It is also stored the address of the filename in which the remaining data related to each ECG is stored. It is used by the application to load the data structures that contain all the information related to the electrocardiographic signal.

\subsection{Results}

Tests has been done in a group of 15 healthy patients included on two age groups: $25-40$ years and $40-60$ years. Five ECG tests were performed to each one.

The reception process was tested when receiving the 75 tests performed by the Cardihogar. This test was performed 10 times and no fatal error occurred from which the application could not recover.

The transmission process was checked sending 15 patients a total of 10 times and no error occurred.

The protection mechanisms for the communication loss where correctly checked by removing the communication wire as a simulation of a connection loss. The system responded as expected without information loss or application blocking.

The database information was consistent during the test procedure and no data was mixed or lost during the tests.

\section{Conclusions}

The main goal of this investigation has been fulfilled because it has been developed an application that makes easier to analyze the ECG information looking forward to prevent cardiac diseases on the primary health level.

The tools used for the development of this solution totally fit the requirements for the application.

The developed solution is an APORTE to the study of cardiac diseases from a preventive focus.

\section{References}

[1] World Health Organization .The World Health Report 2013 at http://www.who.int/researcj/en/.

[2] Park JK. A comparison of Cornell and Sokolow-Lyon Electrocardiographic Criteria for Left Ventricular Hypertrophy in Korean Patients, Korean Circulation Journal, 2012.

[3] Clifford G. Signal processing methods for Heart Rate Variability, University of Oxford, 2002.

[4] Richardson K. Electrocardiographic damage scores and cardiovascular mortality. American Heart Journal, Vol.149, No.3, 2003.

[5] Zayas R. Dispersión del intervalo QT: un predictor de arritmias ventriculares. Revista Cubana de Cardiología y Cirugía Cardiovascular, 2000.

Address for correspondence.

Jorge Aguilera Perez

No. 1704, 202 Street, Siboney 11600, Havana, Cuba

joraguiperez@gmail.com

Rene Gonzalez Fernandez

No. 1704, 202 Street, Siboney 11600, Havana, Cuba

rigf88@gmail.com 